\title{
A mutational analysis of Dictyostelium discoideum multicellular development
}

\author{
Ines Carrin, $\nmid$ Irene Murgia, $\ddagger$ Andrew McLachlan and Robert R. Kay
}

Author for correspondence: Robert R. Kay. Tel: +44 1223 402393. Fax: +44 1223412142.

MRC Laboratory of Molecular Biology, Hills Road, Cambridge CB2 2QH, UK

\begin{abstract}
We have collected Dictyostelium mutants that arrest in development after aggregation, but before first finger formation. A total of 118 mutant strains were isolated and are referred to as mound (mnd) mutants. Nine complementation groups (mndA-mndl), containing 46 of the mutant strains, were defined by parasexual methods. A statistical analysis suggested that there are about 118 genes which, when mutated, confer the mound phenotype. Of these genes, about 60 are predicted to be mutated in our collection: the 9 assigned to complementation groups and another 51 unassigned mutants. mndA, G, $H$ and I were assigned to linkage groups VII, IV, II and VI, respectively. Development of the mutant strains was characterized by terminal morphology, neutral red staining and expression of marker mRNAs for prespore and prestalk cells. Three broad classes were recognized. (1) Postaggregative mutants - those blocked early in multicellular development. They did not express any of the prestalk or prespore marker mRNAs and generally arrested as low mounds or ridges. (2) Pathway mutants - those blocked specifically in either prestalk or prespore differentiation. They expressed either prestalk or prespore marker mRNAs, but not both, and generally proceeded further morphologically than post-aggregative mutants. (3) Morphogenesis mutants - those apparently blocked in morphogenesis rather than cell differentiation. They expressed all the cell-type marker mRNAs tested. Most arrested as tight mounds lacking a tip and of defined upper size, but some mutants produced aberrant tips. The majority of mutants tested synergized with wild-type: 24/28 strains which cannot make spores when developed alone, were able do so when allowed to develop with an equal number of wild-type cells. We suggest that some of the morphogenesis mutants have a cytoskeletal defect which prevents first finger formation and that these mutants can be physically carried through development by the wildtype (synergy by 'piggy-backing').
\end{abstract}

Keywords: Dictyostelium discoideum, development, mound mutants, gene numbers

\section{INTRODUCTION}

Molecular genetic analysis has allowed access to a whole range of master regulatory genes in developmental biology (Horvitz \& Sternberg, 1991; Mayer et al., 1991;

†Present address: Centre for Molecular Biotechnology, Queensland University of Technology, GPO Box 2434, Brisbane Q4001, Australia.

¥Present address: Department of Biology, 'L. Gorini' - University of Milan, via Celoria 26, 20133 Milan, Italy.

Abbreviations: DIF-1, differentiation-inducing factor-1; GBF, G-box binding factor; Ig, linkage group; REMI, restriction enzyme-mediated integration.
Nusslein-Volhard \& Wieschaus, 1980). There are two major prerequisites for its success. Firstly, a genetic system allowing the isolation and maintenance of large numbers of strains carrying mutations which disrupt development. Secondly, some way of cloning the genes so identified. Dictyostelium discoideum has a relatively simple developmental programme which nevertheless displays many of the key phenomena seen in other organisms: cell differentiation, pattern formation, gradients, regeneration and complicated morphogenetic manoeuvres (Bonner, 1967; Loomis, 1982; Raper, 1940).

Since Dictyostelium is haploid and its development facultative, many sorts of mutants blocked in development 
can be readily isolated and maintained. Using parasexual genetics, it is also easy to place them into complementation groups, though the low frequency of recombination is inimical to further genetic analysis (Loomis, 1987; Newell, 1978; Welker \& Williams, 1982). Despite these advantages, the possibilities of genetic analysis have not been greatly exploited because there has been no clear route from mutation to cloned gene.

Recent advances have now made a molecular genetic analysis entirely feasible. The restriction enzyme-mediated integration (REMI) technique for insertional mutagenesis, in which a restriction enzyme targets a transforming plasmid to its recognition sites in the genome, enables genes to be mutated and then readily cloned (Dynes et al., 1994; Harwood et al., 1995; Insall et al., 1994; Kuspa \& Loomis, 1992). Alternatively classical mutants may be complemented using genomic clone banks (Dynes \& Firtel, 1989). Though this method is not routine, it may become so with further improvements in transformation techniques. In our experience, chenical mutagenesis has the advantage that many more mutants can be isolated than by REMI. With these possibilities in mind, we have made a large-scale mutational analysis of early multicellular development in Dictyostelium using chemical mutagenesis.

The period of development analysed starts at the end of aggregation, when the cells have gathered into loose mounds, and ends with the formation of a standing first finger, when an independent, patterned, multicellular entity has been established. In this period, the aggregates first compact and sub-divide into a series of mounds of fixed upper size (Hohl \& Raper, 1964). The final size of a mound is apparently regulated by a series of organizing centres (tips), one per mound, which inhibit each others formation, probably by a graded diffusible signal; we refer to this process as size control (Durston, 1976; Kopachik et al., 1983). The mounds secrete an extracellular matrix, the slime sheath, consisting of cellulose and a number of proteins, including the $\mathrm{ecm} A$ and $\mathrm{ecm} B$ gene products (McRobbie et al., 1988). Within the mounds prestalk and prespore cells differentiate. The prespore cells are relatively homogeneous in both expression of markers and eventual fate (they become spores), but the prestalk cell population is heterogeneous. Prestalk A cells express the $\mathrm{ecm} A \mathrm{mRNA}$ and eventually produce the stalk proper of the mature fruiting body, whereas prestalk $B$ cells express the $\mathrm{emB} \mathrm{mRNA}$ and produce the basal disc (Jermyn et al., 1989; Williams et al., 1989). Prestalk A cells differentiate at scattered positions in the mound and later sort to populate the protruding tip that forms at the top (Williams et al., 1989).

cAMP signalling controls cell movement (Siegert \& Weijer, 1992) and is essential for the continued differentiation of cells after aggregation. Differentiation-inducing factor-1 (DIF-1), a chlorinated alkyl phenone (Kay \& Jermyn, 1983; Morris et al., 1987), induces prestalk and stalk cell differentiation (Town et al., 1976; Williams $t$ t al., 1987). Little is known of its mode of action, though there is a cytoplasmic binding protein (Insall \& Kay, 1990) and
DIF-1 is inactivated by dechlorination, catalysed by DIF1 dechlorinase (Nayler et al., 1992; Traynor \& Kay, 1991). DIF-1 biosynthesis, the binding protein and the metabolic enzymes are developmentally regulated and show a large increase at the end of aggregation.

The aim of our mutational analysis is to eventually identify genes involved in cAMP and DIF-1 signalling, determining the size of an aggregate, cell sorting and morphogenetic movements.

\section{METHODS}

Strains and cell growth. All strains ultimately derive from strain NC4; their genotypes ar.1 origins are shown in Table 1. Strains HM48, HM138 and HM140 are spontaneous cycloheximide-resistant mutants isolated from strain NP2 by selection on plates containing $500 \mu \mathrm{g}$ cycloheximide $\mathrm{ml}^{-1}$. Cells were grown at $22{ }^{\circ} \mathrm{C}$ in association with Klebsiella aerogenes on SM agar plates (Kay, 1987). To initiate development, they were freed from bacteria by repeated centrifugal washes in $\mathrm{KK} 2$ (16.6 $\mathrm{mM} \mathrm{KH}_{2} \mathrm{PO}_{4}, 3.8 \mathrm{mM} \mathrm{K}{ }_{2} \mathrm{HPO}_{4}, 1 \mathrm{mM} \mathrm{MgSO}$, $\mathrm{pH} \mathrm{6.2)}$ and plated on $1.8 \%(\mathrm{w} / \mathrm{v}) \mathrm{L} 28$ agar (Oxoid) containing KK2.

Mutagenesis was performed with NTG (Sigma) at $200 \mu \mathrm{g} \mathrm{ml}^{-1}$ (usually from a $10 \mathrm{mg} \mathrm{ml}^{-1}$ DMSO stock solution) for $20 \mathrm{~min}$, giving a survival of $1-10 \%$ or with UV irradiation (15 s at $302 \mathrm{~nm}$ and $7 \mathrm{~mW} \mathrm{~cm}^{-2}$ ). Cells were plated clonally on SM agar in association with bacteria and colony morphology examined 5-8 d later for mutant development.

Genetics. Diploids were formed by making a mixture of about $10^{6}$ cells (a loop from a growth zone) of each of the two strains to be crossed in $1 \mathrm{ml}$ sterile $20 \mathrm{mM} \mathrm{CaCl}_{2}$ and incubating it in a multiwell plate overnight on an orbital shaker (180 r.p.m.) at $22^{\circ} \mathrm{C}$. Next day the contents of each well were distributed amongst four SM plates and spread in association with bacteria. Diploids were selected at the restrictive temperature of $26.8^{\circ} \mathrm{C}$. Plates were kept in plastic boxes, with a small air hole, to minimize evaporative cooling. Generally each cross gave at least one diploid.

Complementing diploids were immediately obvious from their wild-type fruit and spore head colour (yellow); further confirmation was not normally necessary. Non-complementing diploids were generally segregated to give haploids by growth on thiobendazole (Welker \& Williams, 1980a) $\left(3 \mu \mathrm{g} \mathrm{ml}^{-1}\right.$ in SM agar) and the segregants checked for the presence of outside markers from both parents (normally methanol and cycloheximide resistance).

Mutations were assigned to linkage groups by crossing with the multiply marked tester strain HU407 (from Dr K. Williams, Macquarie University, Australia), segregating haploids and testing their genotypes by standard methods (Loomis, 1987; Newell, 1978).

Mutant strains were stored by freezing vegetative cells in liquid nitrogen and by storing diploid spores, from a cross with a parental strain, on silica gel.

Characterization of mutant strains. Cells were stained with $50 \mu \mathrm{g}$ neutral red $\mathrm{ml}^{-1}$ at the start of development (Bonner, 1952). RNA was extracted from cells that had developed on KK2 agar for different lengths of time and prestalk and prespore mRNAs detected by Northern blotting as before (Berks \& Kay, 1990). In synergy experiments, cells from two different strains were washed free of bacteria and mixed, generally $1: 1$, and plated for development on KK2 agar. The genotype of any spores formed was checked by plating them on SM agar with 
Table 1. Genetically marked strains used in this work

\begin{tabular}{|c|c|c|c|c|c|c|c|c|c|c|c|c|}
\hline \multirow[t]{2}{*}{ Strain } & \multirow[t]{2}{*}{ Parent } & \multicolumn{10}{|c|}{ Genotype* } & \multirow[t]{2}{*}{ Origin } \\
\hline & & $w h i$ & buen & $t s g$ & $c y c$ & acr & axe & $s p r$ & man & bsg & cou & \\
\hline $\mathrm{X} 2$ & & + & $A$ & $A 1$ & + & + & $A 1, B 1$ & + & + & + & + & Williams et al. (1974) \\
\hline $\mathrm{X} 22$ & & $A$ & + & $D 12, E 13$ & + & $A 1$ &,++ & $A 1$ & + & + & + & Williams \& Newell (1976) \\
\hline NP2 & & + & + & $A 1$ & + & + & $A 1, B 1$ & + & + & + & + & Williams et al. (1974) \\
\hline HM48 & HL6 & + & + & $J 2$ & 902 & + & $A 1, B 1$ & + & + & + & + & This study \\
\hline HM138 & NP2 & + & + & $A 1$ & 903 & + & $A 1, B 1$ & + & + & + & + & This study \\
\hline HM140 & NP2 & + & + & $A 1$ & 905 & + & $A 1, B 1$ & + & + & + & + & This study \\
\hline HU407 & & + & + & $K 21$ & $A 1$ & + &,++ & + & $A 2$ & $A 5$ & $A 351$ & Welker \& Williams (1980b) \\
\hline
\end{tabular}

* Phenotypes of mutations at the loci are as follows: whi $A$, white (rather than yellow) spore heads; bwn $A$, brown pigment produced; tsg, grows at $22{ }^{\circ} \mathrm{C}$ but not at $27{ }^{\circ} \mathrm{C}$; acr $A$, grows on $2 \%$ methanol; axe $A$ plus axe $B$, grows axenically; $\operatorname{spr} A$, round (rather than oval) spores; man $A$, lack of $\alpha$-mannosidase; $\operatorname{sgg} A$, unable to grow on Bacillus subtilis; cou $A$, sensitivity to $1.0 \mathrm{mM}$ coumarin.

bacteria, after all non-spores had been killed by detergent treatment $(0 \cdot 3 \%$ Cemulsol for at least $30 \mathrm{~min})$.

\section{RESULTS}

\section{Mutant isolation}

Mutations were induced by NTG or, occasionally, UV mutagenesis. Most mutants were isolated in strains HM140 and X22 (Table 1), which are marked for parasexual genetics and develop well. HM140 is axenic, allowing the possibility of DNA-mediated transformation; other axenic strains (X2, HM48 and HM138; Table 1) were used for a few experiments, but were not so satisfactory. X22 is non-axenic, but was used as the second parent because axenic strains do not cross well with each other in parasexual crosses (Dr P. C. Newell, personal communication, confirmed by our experience).

Mutants were chosen by their plaque morphology when grown on bacterial lawns. Wild-type plaques display the entire developmental sequence from growing cells around the edge of the plaque, to fruiting bodies in the centre. Mutants blocked at various stages of development are readily recognized; we chose ones which aggregated, but then arrested as mounds or ridges, in some cases with a tip. Because the common feature of the chosen mutants was their arrest in development at the mound stage, we refer to them as mound (mnd) mutants. Mutants that proceeded further and made a slug were not taken, nor were ones with a leaky phenotype. A total of 118 mutants were collected from more than 100 separate mutagenesis experiments in which about 200000 plaques were observed.

\section{Complementation testing}

Dictyostelium is a naturally haploid organism in which parasexual genetics depends on the rare fusion of two cells to form a stable diploid. Diploids were selected by using parental strains with complementing temperature-sensitive growth mutations and selecting at the restrictive temperature (Katz \& Sussman, 1972; Loomis, 1987;

\section{Table 2. Complementation groups}

Mutants raised in an axenic background were crossed with those raised in a non-axenic background. The parent of all non-axenic strains is X22; of the axenic strains, X2 is the parent of strains HM51-HM90, HM48 is the parent of HM91, NP2 is the parent of HM142 and HM144, HM138 is the parent of HM153 and HM140 is the parent of the rest.

\begin{tabular}{|lll|}
\hline & Axenic strains & Non-axenic strains \\
\hline$m n d A$ & HM57 HM142 & HM98 HM120 \\
& HM61 HM153 & HM108 HM123 \\
& HM62 HM158 & HM109 HM128 \\
& HM72 HM160 & HM110 HM224 \\
& HM73 HM183 & \\
& HM79 HM202 & \\
& HM91 HM203 & \\
$m n d B$ & NP127 & HM113 HM 137 \\
& & HM114 \\
$m n d C$ & HM52 HM205 & HM133 \\
& HM56 & \\
$m n d D$ & HM59 & HM130 \\
$m n d E$ & HM81 & HM157 \\
$m n d F$ & HM144 & HM161 \\
$m n d G$ & HM173 HM208 & HM122 HM228 \\
& & HM135 \\
$m n d H$ & HM155 HM182 & HM212 \\
$m n d I$ & HM148 & HM213 \\
\hline
\end{tabular}

Newell, 1978). All mutants were first crossed with a parental strain to test for dominance. All were recessive except for the mutations in strains HM74 and HM226, which were not used further.

Essentially, all mutants in the non-axenic parent, strain $\mathrm{X} 22$, were crossed with the mutants in the axenic background (strains HM140, HM48, HM138, HM140, $\mathrm{X} 2)$ in a $59 \times 57$ matrix. Once it was established whether or not a mutant belonged to a particular complementation group, further crosses with other members of the group 


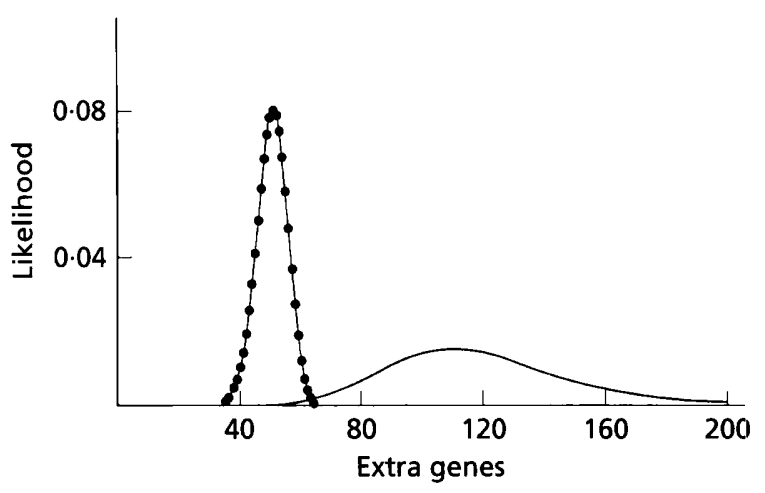

Fig. 1. Statistical estimate of the number of genes essential for development of a loose aggregate into a first finger. This estimate is based on the frequency of non-complementing diploids in almost 2000 crosses between the mutants and takes into account the observed differences in mutation frequency between loci. The broad curve without symbols gives the likelihood of different numbers of additional genes, over the 9 already established (i.e. assigned to complementation groups). It peaks at 109 extra genes and, with the 9 established genes, this gives 118 as the likeliest number of $m$ nd genes. Of the 109 extra genes, some will be already present in our mutant collection (in one parent, but not in both) and the likelihood plot for this number is shown in the second curve (O): it is most likely to be 51 .

were not generally made. Overall, almost 2000 crosses were made. A few diploids could not be isolated after several attempts and crosses with a few strains were discontinued for various reasons; in the statistical analysis it is assumed that these crosses would all have given complementing diploids. To the extent that this assumption is wrong, we will have slightly over-estimated the number of target genes giving the mound phenotype. The diploids either developed as wild-type or mutant, intermediate forms were not generally seen. Thus a clear pattern of complementation and non-complementation was apparent and nine complementation groups were established (Table 2). Of the 116 mutants analysed by complementation, $46(40 \%)$ were placed in complementation groups. mnd $A$ is a notable mutational hotspot with 22 alleles recovered, compared to five in the next most populous complementation group.

\section{Estimate of gene numbers}

A statistical estimate, using a new method developed by Andrew McLachlan (from whom the computer program may be obtained on request at the above address), suggested that the total number of genes giving the mound phenotype was most likely to be 118 (Fig. 1). This method utilizes the observed spectrum of mutation frequencies at individual loci to estimate the likeliest number of such loci. Unlike earlier methods, which had to assume equal mutation frequencies at all loci (Coukell, 1975; Warren et al., 1975), it is able to accommodate mutational hotspots such as $m n d A$. From the complementation analysis, nine loci have been established; Fig. 1 shows that there are likely to be an additional 109 loci, not yet assigned to complementation groups, making 118 loci in all. Of the extra loci, we estimate that 51 are represented in one parent or the other (but not both). Thus, we may have isolated mutants in about half of the genes giving the mound phenotype.

\section{Linkage analysis}

Mutations in four of the complementation groups were assigned to linkage groups after crossing with the multiply marked tester strain, HU407 (Welker \& Williams, 1980b; Table 1). The strains used (HM98, HM235, HM212 and HM213) all derive from the strain X22 parent and, when crossed with strain HU407, give diploids having all six established linkage groups marked. Haploids were segregated and cloned from each of the resulting diploids, and their genotypes determined (Table 3). Coumarin sensitivity $(\operatorname{cou} A)$, the standard marker for linkage group VII (lgVII) (Welker \& Williams, 1980b) proved very hard

\section{Table 3. Assignment to linkage groups}

Mutant strains (HM98, HM135, HM212 and HM213) were crossed with the multiply marked tester strain HU407, the resulting diploids haploidized and the genotypes of the resulting segregants determined.

\begin{tabular}{|c|c|c|c|c|c|c|c|c|c|c|c|c|}
\hline & \multicolumn{2}{|l|}{ I } & \multicolumn{2}{|l|}{ II } & \multicolumn{2}{|c|}{ III } & \multicolumn{2}{|l|}{ IV } & \multicolumn{2}{|l|}{ VI } & \multicolumn{2}{|l|}{ VII } \\
\hline & $c y c A I$ & + & $\operatorname{acr} A 1$ & + & bsgA & + & bwnA1 & + & $\operatorname{man} A 2$ & + & $t \operatorname{sg} K 21+\operatorname{cou} A$ & + \\
\hline$m n d A$ & 10 & 13 & 9 & 14 & 8 & 15 & 4 & 19 & 14 & 9 & 3 & 0 \\
\hline+ & 16 & 11 & 12 & 15 & 11 & 16 & 8 & 19 & 13 & 14 & 0 & 4 \\
\hline$m n d G$ & 16 & 16 & 13 & 19 & 8 & 24 & 0 & 32 & 11 & 21 & 14 & 18 \\
\hline+ & 5 & 9 & 7 & 7 & 6 & 8 & 14 & 0 & 6 & 8 & 11 & 3 \\
\hline$m n d H$ & 29 & 9 & 38 & 0 & 24 & 14 & 11 & 27 & 18 & 20 & 27 & 11 \\
\hline+ & 2 & 9 & 0 & 11 & 2 & 9 & 2 & 9 & 1 & 10 & 2 & 9 \\
\hline$m n d I$ & 6 & 13 & 17 & 2 & 6 & 13 & 2 & 17 & 0 & 19 & 10 & 9 \\
\hline+ & 9 & 10 & 19 & 0 & 17 & 2 & 19 & 0 & 19 & 0 & 18 & 1 \\
\hline
\end{tabular}



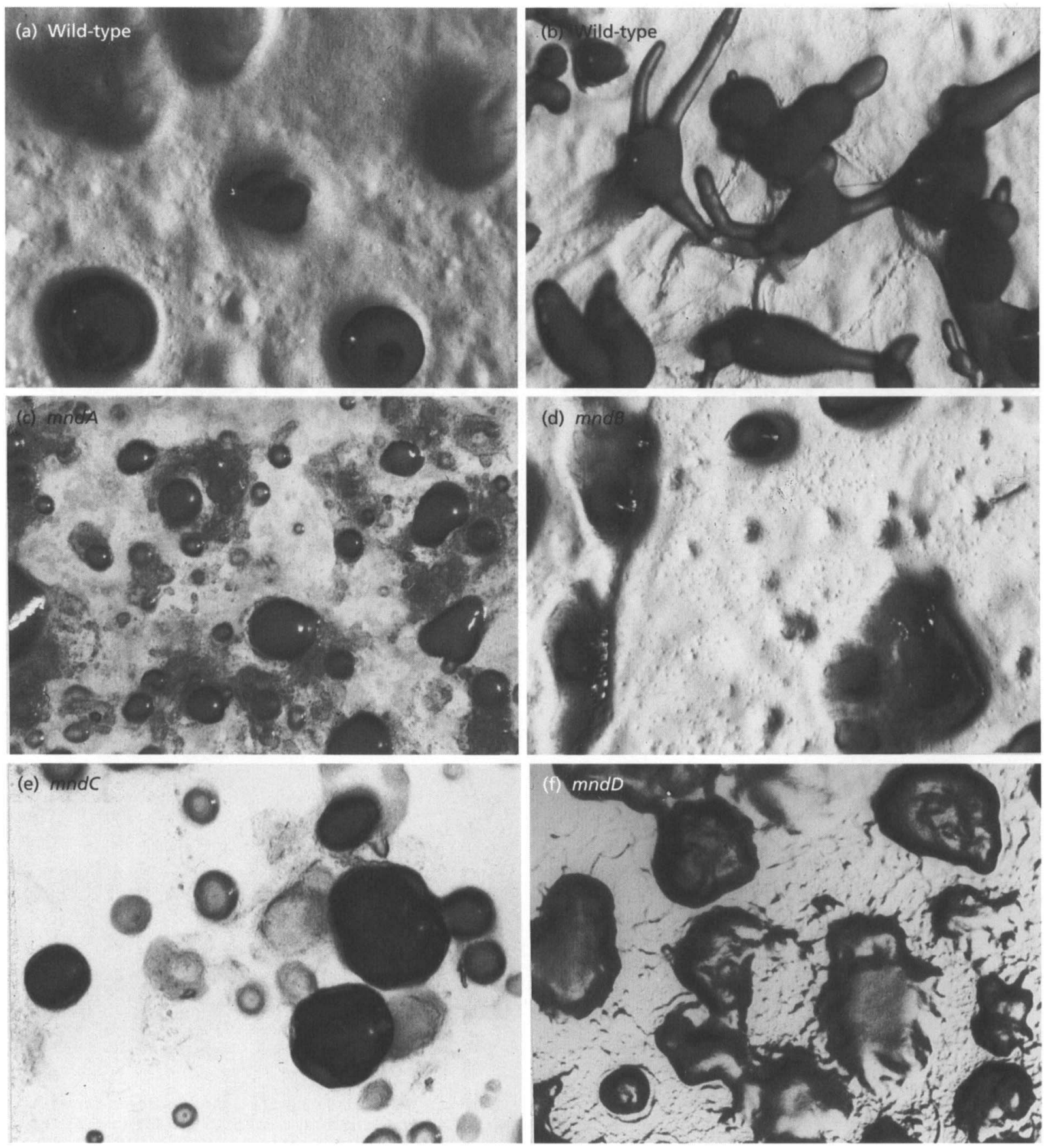

Fig. 2. Morphology of wild-type and mutants. All photographs are of stock plates of cells grown on SM agar in association with $K$. aerogenes and are taken from above (all $\times 45)$. Terminal mutant phenotypes are shown. (a) Strain X22 (wild-type parent), mounds with tips just emerging; (b) strain X22, first fingers forming; (c) strain HM183 (mndA), tight mounds, no tip; (d) strain NP127 (mndB), mounds less tight than mndA, no tip; (e) strain HM205 (mndC), raised mounds, no clear tip; $(f)$ strain HM130 (mndD), interconnected, irregular mounds, no tip.

to score. Instead, $t \mathrm{sg} K$ was used as the $\lg$ VII marker, after first eliminating strains having $t s g D$ or $t s g E$ by the linkage of these markers to $c y c A$ and acr $A$, respectively. Some of the segregation patterns of unlinked markers are strongly biased, occasionally with a whole class of expected segregant missing (as in $m n d I$ ). This problem is frequently encountered in parasexual crosses and may be due to the presence of deleterious, linked mutations (Newell, 1978; Newell et al., 1977; Welker \& Williams, 1980b). Nevertheless, Table 3 shows that in each cross, the mound mutation segregated independently of markers in five of the six linkage groups but was linked to the remaining marker. Thus, mnd $A$ was assigned to $\operatorname{lgVII}$, mndG to $\operatorname{lgIV}, m n d H$ to $\lg I \mathrm{I}$ and $m n d I$ to $\lg \mathrm{VI}$ (with a little less 

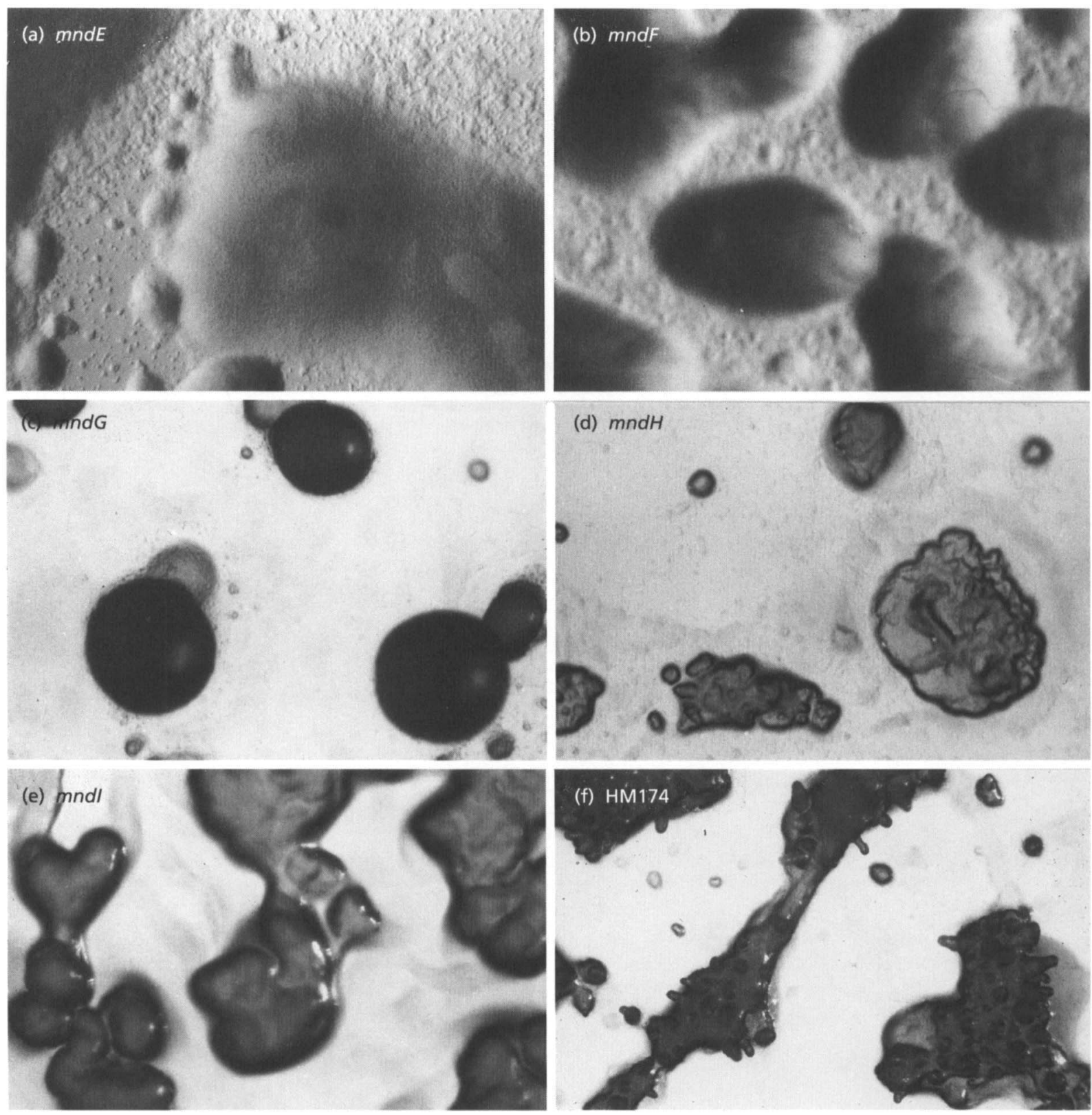

Fig. 3. Morphology of mutants. All photographs are of stock plates of cells grown on SM agar in association with $K$. aerogenes and are taken from above (all $\times 45)$. Terminal mutant phenotypes are shown. (a) Strain HM81 (mndE), large, low mounds, no tip; (b) strain HM161 ( $m n d F)$, mounds, sometimes with a tip-like protrusion; (c) strain HM208 ( $m n d G$ ) compact, roughly hemispherical mounds, no tip; (d) strain HM212 (mndH), scalloped ('brainy') mounds, no definite tip; (e) strain HM213 ( $m n d l)$, irregular, interconnected mounds, no tip; $(f)$ strain HM174 (unassigned), extensive mounds with multiple, arrested tips.

confidence due to the biased segregation of some markers).

\section{Morphological classification}

Many, though not all, of the mutants can be regarded as arrested stages in the wild-type sequence of morphological development. We, therefore, based our morphological classification on the wild-type sequence as seen in clones or streaks of cells growing and developing on SM agar, in association with bacteria (Figs 2 and 3, Table 4).
Morphological class 1 mutants. These arrest shortly after aggregation as low, loose mounds. There is no evidence for an upper fixed size for the aggregates or for a slime sheath.

Morphological class 2 mutants. These produce more tightly packed aggregates, which may have scalloped surfaces $(m n d B)$ and appear sub-divided, indicating that there is some sort of size control. A slime sheath is not normally present.

Morphological class 3 mutants. These produce discrete 
Table 4. Phenotypes of mutants assigned to complementation groups

\begin{tabular}{|c|c|c|c|c|c|c|c|c|}
\hline \multirow{2}{*}{$\begin{array}{l}\text { Complementation } \\
\text { n group }\end{array}$} & \multirow[t]{2}{*}{ Example } & \multirow{2}{*}{$\begin{array}{c}\text { Morphology* } \\
y\end{array}$} & \multicolumn{3}{|c|}{ mRNA expression $\dagger$} & \multicolumn{2}{|c|}{ Spores $\ddagger$} & \multirow{2}{*}{$\begin{array}{c}\text { Neutral red } \\
\text { staining } \$\end{array}$} \\
\hline & & & $e c m A$ & $e c m B$ & $p s A$ & Alone & Synergy & \\
\hline \multirow[t]{3}{*}{$m n d A$} & HM79 & 3 & ++ & $+t$ & ++ & - & + & Red eye \\
\hline & HМ98 & 3 & ++ & + & ++ & - & + & Red eye \\
\hline & HM142 & 3 & $(+)$ & $(+)$ & + & & & \\
\hline \multirow[t]{4}{*}{$m n d B$} & NP127 & 2 & $(+)$ & ++ & +++ & - & + & \\
\hline & HM113 & 2 & $(+)$ & + & ++ & - & + & Sectors \\
\hline & HM114 & 2 & $(+)$ & + & ++ & + & + & Sectors \\
\hline & HM137 & 2 & $(+)$ & ++ & ++ & + & + & Sectors \\
\hline \multirow[t]{2}{*}{$m n d C$} & HM52 & 4 & + & + & +++ & - & + & Uniform \\
\hline & HM56 & 4 & ++ & ++ & ++ & - & + & Uniform \\
\hline \multirow[t]{2}{*}{$m n d D$} & HM59 & 2 & 0 & + & 0 & - & $(+)$ & Uniform \\
\hline & HM130 & 2 & 0 & 0 & 0 & - & $(+)$ & \\
\hline \multirow[t]{2}{*}{$m n d E$} & HM81 & 1 & 0 & + & 0 & - & $(+)$ & Uniform \\
\hline & HM157 & 1 & 0 & + & 0 & - & $(+)$ & \\
\hline \multirow[t]{2}{*}{$m n d F$} & HM144 & 2 & $(+)$ & ++ & 0 & - & + & \\
\hline & HM161 & 2 & + & + & 0 & & & \\
\hline \multirow[t]{4}{*}{$m n d G$} & HM135 & 3 & +++ & +++ & +++ & & & \\
\hline & HM173 & 3 & +++ & + & +++ & & & \\
\hline & HM208 & 3 & +++ & +++ & +++ & & & \\
\hline & HM228 & 3 & +++ & +++ & +++ & & & \\
\hline \multirow[t]{2}{*}{$m n d H$} & HM155 & 2 & $(+)$ & + & +++ & - & + & \\
\hline & HM212 & 2 & $(+)$ & + & ++ & & & \\
\hline \multirow[t]{2}{*}{$m n d I$} & HM148 & 2 & ++ & ++ & ++ & - & + & Uniform \\
\hline & HM213 & 2 & + & + & +++ & & & \\
\hline
\end{tabular}

* The morphological classes are explained in the text

$\dagger$ Expression of the prestalk genes $e \mathrm{~cm} A$ and $e \mathrm{~cm} B$ and the prespore gene $p s A$ was determined by Northern blotting; expression levels of the mutants at $21 \mathrm{~h}$ were compared to the parental strains after $15 \mathrm{~h}$ of development. Approximate levels are as follows:,+++ control; ++ , $20-50 \%$ of control; + , detectable, but less than $20 \%$ of control; $(+)$, trace; 0 not detectable.

$\ddagger$ Spore formation is indicated with the mutant cells developing alone or in synergy with equal numbers of wild-type cells. Synergy is scored as follows: + , mutant produced approximately the same number of spores in the synergy mixture as did the wild-type; $(+)$, mutant produced less than $5 \%$ wild-type spore yield; - , mutant produced no spores.

$\int$ Red-eye staining is a red central core, normally at the top of the mound; sector staining is staining of cells at the boundaries of sectors in large aggregates; uniform staining is as the name suggests.

mounds, roughly hemispherical or raised to resemble tree trunks, containing tightly packed cells. There is definite size control: large aggregates are visibly sub-divided into mounds of at most $0.5 \mathrm{~mm}$ diameter (though some X22derived strains produce mounds of up to $1.5 \mathrm{~mm}$ diameter). A slime sheath is formed, as evidenced by a shiny surface, but there is no sign of a tip.

Morphological class 4 mutants. This is a more heterogeneous class, the common property being tip formation. Some strains have large aggregates, divided into sectors each having a tip-like protrusion; at the other extreme, single, compact mounds with tips are produced.

\section{Neutral red staining}

We used neutral red as a convenient way of detecting the differentiation and patterning of prestalk cells during development of the mutants. Previous work showed that neutral red stains the prestalk cells in the anterior of the slug (Bonner, 1952) and a scattering of 'anterior-like' cells in the posterior, but does not stain the prespore cells (Sternfeld \& David, 1981). However, we observed a complicated staining pattern that evolves in the mound prior to tip and slug formation (Carrin, 1990). During aggregation of the wild-type, all cells stain evenly with neutral red; distinct, more strongly staining cells do not appear until the low mound stage. They first appear at scattered positions in the mound but then gather into a central 'red-eye' at the top, before dispersing again, usually down the surface of the mound. A tip forms a little after the red-eye has dispersed. We assume that this staining reflects the appearance and movement of a class of prestalk cells before tip formation. Various of the mound mutants arrest at particular stages in the process. In $m n d C, D$ and $E$ strains, neutral red-stained cells are uniformly distributed in the aggregate, in $m n d B$ strains 

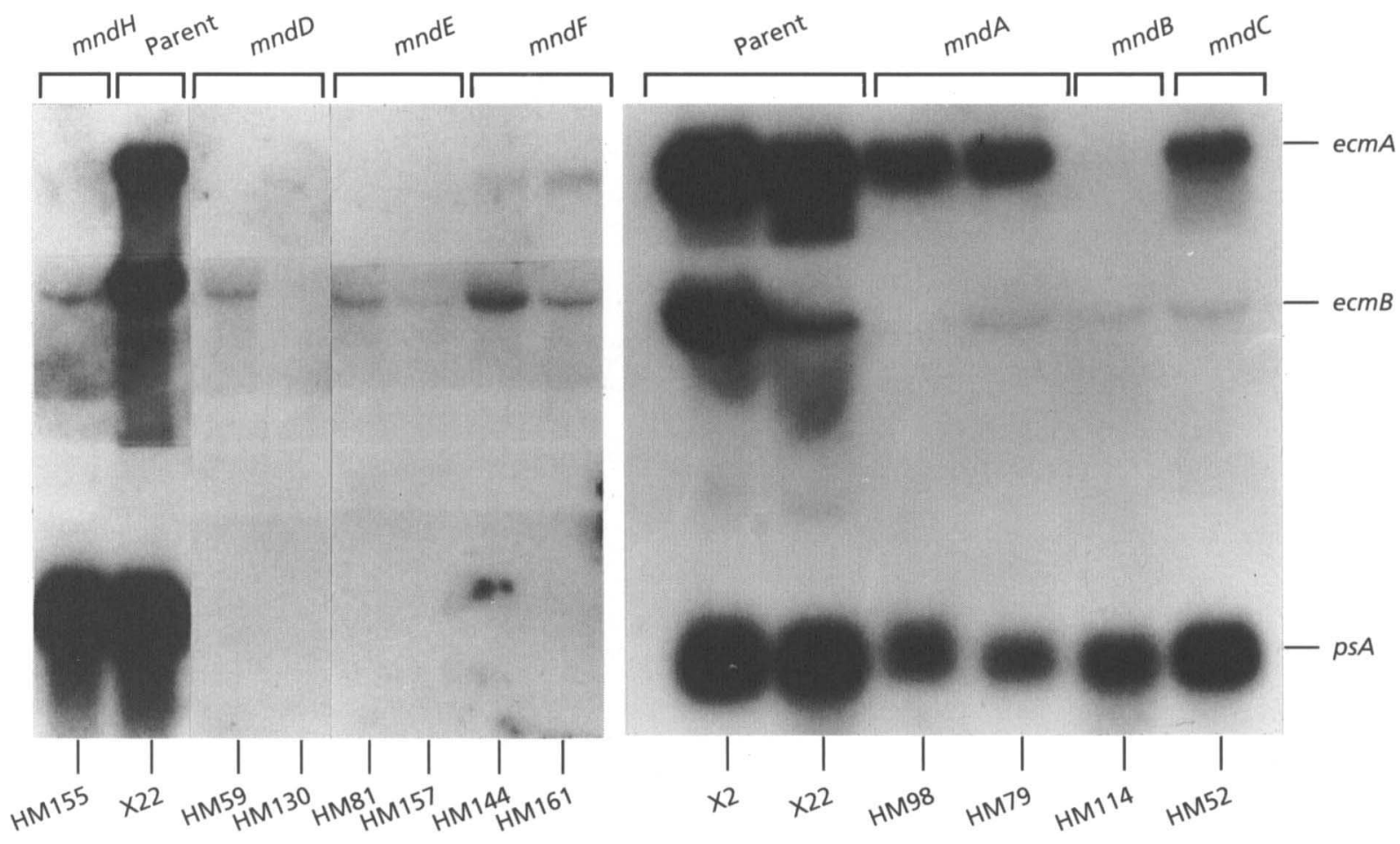

Fig. 4. Gene expression by selected mutants. Strains were allowed to develop on KK2 agar for $21 \mathrm{~h}$ (parental strains are shown at $15 \mathrm{~h}$ ), RNA extracted and $5 \mu \mathrm{g}$ per lane analysed in a Northern blot. Prestalk A cells expressed ecmA mRNA, prestalk $B$ cells expressed ecmB mRNA (and possibly ecmA mRNA) and prespore cells expressed psA mRNA. Expression levels were nearly always lower in the mutants than in the wild-type, but clear differences were apparent between them. For instance $m n d A$ mutants clearly expressed ecmA and ps $A$ and some alleles also expressed ecmB, whereas $m n d B$ mutants did not express ecmA and $m n d E$ mutants expressed only ecmB and this with only low efficiency.

sectors form with stained cells at the borders and in mnd $A$ there is a very prominent red-eye (Table 4).

\section{Prestalk and prespore differentiation}

Specific mRNAs were used to monitor the differentiation of prespore and prestalk cells in the mutants. The ps $A$ mRNA (Barklis \& Lodish, 1983; Early et al., 1988) encodes a cell-surface protein of unknown function which is expressed in all prespore cells. ecm $A$ and $e c m B$ mRNAs are specific markers for prestalk cells and both encode proteins of the extracellular matrix (i.e. the slime sheath). Their expression patterns are complicated and define several subclasses of prestalk cell (Ceccarelli et al., 1991; Early et al., 1993; Jermyn et al., 1987). Both ecm $A$ and $e c m B$ expression is induced by DIF-1 and expression of these markers therefore indicates that cells both produce and respond to DIF-1. Expression of all three markers commences in wild-type strains 1-2 $\mathrm{h}$ before tip formation and increases rapidly during first finger extension (Berks \& Kay, 1990; Jermyn et al., 1987). Cells were allowed to develop on non-nutrient agar and harvested after 15-17 h, the time when the parental strains would be making tips, and at $20-21 \mathrm{~h}$, to allow for the possibility of slow development in the mutant strains. The RNA (Northern) blots revealed three very general classes of mutants, which correlated quite well with the morphological classification (Fig. 4, Table 4).
1. Post-aggregative block mutants (e.g. $m n d E$ ), in which there was no, or very little, expression of prespore or prestalk mRNAs. These were generally in morphological class 1 and we assume that they have a general block to post-aggregative development.

2. Pathway mutants (e.g. $m n d B, F$ and $H$ ), in which there was expression of one or two cell-type-specific mRNAs but not of the remaining one(s). These mutants were generally in morphological class 2 and we assume that they have blocks in one or more specific pathways of cell differentiation.

3. Morphogenesis mutants (e.g. mnd $A, C, G \& I$ ), in which all three cell types differentiate. These mutants are generally in morphological classes 3 and 4 . One possible explanation of their failure to develop is that they are defective in some morphogenetic process necessary for tip formation and elongation (see Discussion).

\section{Synergy with wild-type}

Many of the mutants were tested for synergy with wildtype. Equal numbers of mutant and wild-type cells were mixed and allowed to develop on non-nutrient agar. Spores were harvested from the resulting fruiting bodies and plated clonally to determine their genotype. Surprisingly, 24 out of 28 of our mutants could form 
spores in synergy with wild-type, but not alone (see Table 4). Because stalk cells are dead, we were not able to determine the genotype of the stalk cells in synergizing mixtures and so are unsure whether synergizing strains could form stalk cells as well as spores.

\section{DISCUSSION}

We have reported the first large-scale hunt for mutants blocked in the multicellular stages of Dictyostelium development. Previous work has either concentrated on the earlier aggregation stage (Coukell, 1975; Darmon $e t$ al., 1977; Williams \& Newell, 1976), or sought more specific classes of mutants, such as the sluggers (Newell \& Ross, 1982), rapidly developing (Abe \& Yanagisawa, 1983; Kessin, 1977) or stalky mutants (Morrissey \& Loomis, 1981).

Statistical analysis suggests that about 118 genes are essential for the transition from a rather amorphous aggregate of cells to a series of separate unitary organisms, each with differentiated cells organized in a discrete spatial pattern and capable of complicated morphogenetic movements. More genes must be involved; those that are also essential for growth or block development at an earlier stage would not have been recovered, nor would mutations in important, but non-essential genes. Our estimate is comparable to that of Loomis, who compared mutation frequencies at known single loci with frequencies of various developmental mutants recovered in the same experiment. This suggested that about 150 genes are essential for aggregation and 300 for development as a whole (Loomis, 1978).

The mutants can be classified into three broad groups, based on terminal morphology and patterns of gene expression. This scheme is not definitive, but should assist in recognizing particular types of mutant.

Post-aggregative mutants arrest development as loose mounds and do not express prestalk or prespore mRNA markers, nor are prestalk cells detectable by neutral red staining. These mutants are very similar to the G-box binding factor (GBF) null mutant (Schnitzler et al., 1994). The GBF protein is a DNA binding protein with sites in the promoters of many post-aggregative genes and these genes are poorly expressed in the GBF null mutant. The GBF protein is activated by extracellular cAMP signals using a transduction pathway distinct from the one used to activate gene expression during aggregation (Schnitzler et al., 1995). Genes such as $m n d D$ and $m n d E$ may encode components of this transduction pathway, or other functions necessary for driving gene expression after aggregation.

The pathway mutants arrest as more compact mounds, though lacking a tip, and express either prestalk or prespore mRNAs but not both. They may therefore define genes which are directly involved in specifying cell fate. $m n d H$ cells express the prespore marker psA well, but the prestalk markers $e \mathrm{~cm} A$ and $e \mathrm{~cm} B$ poorly. Since $e \mathrm{~cm} A$ and $e c m B$ expression is induced by DIF- 1 and the $m n d H$ cells do produce DIF-1 during development (unpublished observations), they may be deficient in their response to DIF-1. Conversely, $m n d F$ cells appear to have a specific defect in prespore differentiation.

The morphogenesis mutants arrest as tight mounds, possibly with a rudimentary tip and express both prestalk and prespore markers. In the best studied example, mnd $A$, neutral red staining shows that prestalk cells arise and sort to the top of the mound. These mutants raise a conundrum, since there is no obvious defect in gene expression to explain their failure to form a first finger. Cytoskeletal mutants, such as those in myosin $2(m b c A)$ and multiple actin-binding proteins, have a similar phenotype to $m n d A$, also expressing prestalk and prespore genes but arresting as tip-less mounds (De Lozanne \& Spudich, 1987; Witke et al., 1992). Evidently, elongation of the mound into a first finger imposes special demands on the cytoskeleton, which are not so apparent at other stages of the life-cycle. A further similarity is that both $m n d A$ and $m b c A$ mutants can synergize well with the wild-type (unpublished observations). Thus, we propose that mutants such as $m n d A$ are defective in morphogenesis and that they may have defects in the cytoskeleton. However, $m n d A$ is not identical to $m b c A$ as they map to different linkage groups [ $m n d A$ to $\lg$ VII and $m b c A$ to $\operatorname{lgIV}$ (Welker et al., 1989)].

The majority of mutants synergized with wild-type to form spores. Synergy is normally assumed to result from the wild-type providing the mutant with some essential signal which it cannot make for itself. Such synergy is relatively rare in other organisms. But another type of synergy is also conceivable in which mutants with a defective cytoskeleton are physically carried through morphogenesis by the wild-type ('piggy-backing'). Piggy-backing is the likeliest explanation for the synergy of the myosin 2 null mutant. The relative abundance of piggy-backing mutants in our collection may contribute to the high overall frequency of synergy.

\section{REFERENCES}

Abe, K. \& Yanagisawa, K. (1983). A new class of rapidly developing mutants in Dictyostelium discoideum: implications for cyclic AMP metabolism and cell differentiation. Dev Biol 95, 200-210.

Barklis, E. \& Lodish, H. F. (1983). Regulation of Dictyostelium discoideum mRNAs specific for prespore or prestalk cells. Cell 32, 1139-1148.

Berks, M. \& Kay, R. R. (1990). Combinatorial control of cell differentiation by cAMP and DIF-1 during development of Dictyostelium discoideum. Development 110, 977-984.

Bonner, J. T. (1952). The pattern of differentiation in amoeboid slime molds. Am Nat 86, 79-89.

Bonner, J. T. (1967). The Cellular Slime Molds. Princeton, $\mathrm{NJ}$ : Princeton University Press.

Carrin, M. I. (1990). A mutational analysis of cell-differentiation and morphogenesis during Dictyostelium discoideum development. $\mathrm{PhD}$ Thesis, University of Cambridge.

Ceccarelli, A., Mahbubani, H. \& Williams, J. G. (1991). Positively and negatively acting signals regulating stalk cell and anterior-like cell differentiation in Dictyostelium. Cell 65, 983-989.

Coukell, M. B. (1975). Parasexual genetic analysis of aggregation- 
deficient mutants of Dictyostelium discoideum. Mol Gen Genet 142, 119-135.

Darmon, M., Barrand, P., Brachet, P., Klein, C. \& Pereira Da Silva, L. (1977). Phenotypic suppression of morphogenetic mutants of Dictyostelium discoideum. Dev Biol 58, 174-178.

De Lozanne, A. \& Spudich, J. A. (1987). Disruption of the Dictyostelium myosin heavy chain gene by homologous recombination. Science 236, 1086-1091.

Durston, A. J. (1976). Tip formation is regulated by an inhibitory gradient in the Dictyostelium discoideum slug. Nature 263, 126-129.

Dynes, J. L. \& Firtel, R. A. (1989). Molecular complementation of a genetic marker in Dictyostelium using a genomic DNA library. Proc Natl Acad Sci USA 86, 7966-7970.

Dynes, J. L., Clark, A. M., Shaulsky, G., Kuspa, A., Loomis, W. F. \& Firtel, R. A. (1994). $\mathrm{LagC}$ is required for cell-cell interactions that are essential for cell-type differentiation in Dictyostelium. Genes Dev $\mathbf{8}$, 948-958.

Early, A., Williams, J., Meyer, H., Por, S., Smith, E., Williams, K. \& Gooley, A. (1988). Structural characterization of Dictyostelium discoideum prespore-specific gene $\mathrm{D} 19$ and of its product, cell surface glycoprotein psA. Mol Cell Biol 8, 3458-3466.

Early, A. E., Gaskell, M. J., Traynor, D. \& Williams, J. G. (1993). Two distinct populations of prestalk cells within the tip of the migratory Dictyostelium slug with differing fates a culmination. Development 118, 353-362.

Harwood, A. J., Plyte, S. E., Woodgett, J., Strutt, H. \& Kay, R. R. (1995). Glycogen synthase kinase 3 regulates cell fate in Dictyostelium. Cell 80, 139-148.

Hohl, H. \& Raper, K. (1964). Control of sorocarp size in the cellular slime mold Dictyostelium discoideum. Dev Biol 9, 137-153.

Horvitz, H. R. \& Sternberg, P. W. (1991). Multiple intercellular signalling systems control the development of the Caenorbabditis elegans vulva. Nature 351, 535-541.

Insall, R. H. \& Kay, R. R. (1990). A specific DIF binding protein in Dictyostelium. EMBO J 9, 3323-3328.

Insall, R. H., Kuspa, A., Lilly, P. J., Shaulsky, G., Levin, L. R., Loomis, W. F. \& Devreotes, P. (1994). CR AC, a cytosolic protein containing a pleckstrin homology domain, is required for receptor and G-protein mediated activation of adenylyl cyclase in Dictyostelium. J Cell Biol 126, 1537-1545.

Jermyn, K. A., Berks, M., Kay, R. R. \& Williams, J. G. (1987). 'Two distinct classes of prestalk-enriched messenger RNA sequences in Dictyostelium discoideum. Development 100, 745-755.

Jermyn, K. A., Duffy, K. T. I. \& Williams, J. G. (1989). A new anatomy of the prestalk zone in Dictyostelium. Nature 340, 144-146.

Katz, E. \& Sussman, M. (1972). Parasexual recombination in Dictyostelium discoideum: selection of stable diploid heterozygotes and stable haploid segregants. Proc Natl Acad Sci US A 69, 495-498.

Kay, R. R. (1987). Cell differentiation in monolayers and the investigation of slime mold morphogens. Methods Cell Bioi 28, 433-448.

Kay, R. R. \& Jermyn, K. A. (1983). A possible morphogen controlling differentiation in Dictyostelium. Nature 303, 242-24.4.

Kessin, R. H. (1977). Mutations causing rapid development of Dictyostelium discoideum. Cell 10, 703-708.

Kopachik, W., Oohata, A., Dhokia, B., Brookman, J. J. \& Kay, R. R. (1983). Dictyostelium mutants lacking DIF, a putative morphogen. Cell 33, 397-403.

Kuspa, A. \& Loomis, W. F. (1992). Tagging developmental genes in Dictyostelium by restriction enzyme mediated integration of plasmid DNA. Proc Natl Acad Sci USA 89, 8803-8807.
Loomis, W. F. (1978). The number of developmental genes in Dictyostelium discoideum. In The Molecular Basis of Cell-Cell Interaction, pp. 497-505. Edited by R. A. Lerner \& D. Bergsma. New York: Liss.

Loomis, W. F. (1982). The Development of Dictyostelium discoideum. New York: Academic Press.

Loomis, W. F. (1987). Genetic tools for Dictyostelium discoideum. Methods Cell Biol 28, 31-65.

McRobbie, S. J., Tilly, R., Blight, K., Ceccarelli, A. \& Williams, J. G. (1988). Identification and localization of proteins encoded by two DIF inducible genes of Dictyostelium. Dev Biol 125, 59-63.

Mayer, U., Torres, R. A., Berleth, T., Misera, S. \& Jurgens, G. (1991). Mutations affecting body organization in the Arabidopsis embryo. Nature 353, 402-407.

Morris, H. R., Taylor, G. W., Masento, M. S., Jermyn, K. A. \& Kay, R. R. (1987). Chemical structure of the morphogen differentiation inducing factor from Dictyostelium discoideum. Nature 328, 811-814.

Morrissey, J. H. \& Loomis, W. F. (1981). Parasexual genetic analysis of cell proportioning mutants of Dictyostelium discoideum. Genetics $\mathbf{9 9}$, 183-196.

Nayler, O., Insall, R. \& Kay, R. R. (1992). Differentiation-inducingfactor dechlorinase, a novel cytosolic dechlorinating enzyme from Dictyostelium discoideum. Eur J Biochem 208, 531-536.

Newell, P. C. (1978). Genetics of the cellular slime molds. Annu Rev Genet 12, 69-93.

Newell, P. C. \& Ross, F. M. (1982). Genetic analysis of the slug stage of Dictyostelium discoideum. J Gen Microbiol 128, 1639-1652.

Newell, P. C., Ratner, D. I. \& Wright, D. (1977). New techniques for cell fusion and linkage analysis of Dictyostelium discoideum. In Development and Differentiation in the Cellular Slime Moulds, pp. 51-61. Edited by P. Cappuccinelli \& J. M. Ashworth. Amsterdam: Elsevier.

Nusslein-Volhard, C. \& Wieschaus, E. (1980). Mutations affecting segment number and polarity in Drosopbila. Nature 287, 795-801.

Raper, K. B. (1940). Pseudoplasmodium formation and organisation in Dictyostelium discoideum. J Elisha Mitchell $S_{c i} S_{o c} \mathbf{5 6}$, 241-282.

Schnitzler, G. R., Fischer, W. H. \& Firtel, R. A. (1994). Cloning and characterization of the G-box binding factor, an essential component of the developmental switch between early and late development in Dictyostelium. Genes Dev 8, 502-514.

Schnitzler, G. R., Briscoe, C., Brown, J. M. \& Firtel, R. A. (1995). Serpentine cAMP receptors may act through a $G$ proteinindependent pathway to induce post-aggregative development in Dictyostelium. Cell 81, 737-745.

Siegert, F. \& Weijer, C. J. (1992). Three-dimensional scroll waves organize Dictyostelium slugs. Proc Natl Acad Sci US A 89, 6433-6437.

Sternfeld, J. \& David, C. N. (1981). Cell sorting during patternformation in Dictyostelium. Differentiation 20, 10-21.

Town, C. D., Gross, J. D. \& Kay, R. R. (1976). Cell differentiation without morphogenesis in Dictyostelium discoideum. Nature 262, 717-719.

Traynor, D. \& Kay, R. R. (1991). The DIF-1 signalling system in Dictyostelium: metabolism of the signal. J Biol Chem 266, 5291-5297.

Warren, A. J., Warren, W. D. \& Cox, E. C. (1975). Genetic complexity of aggregation in the cellular slime mold Polysphondylium violaceum. Proc Natl Acad Si US A 72, 1041-1042.

Welker, D. L. \& Williams, K. (1980a). Mitotic arrest and chromosome doubling using thiabendazole, cambendazole, nocodazole and ben late in the slime mould Dictyostelium discoideum. $J$ Gen Microbiol 116, 397-407. 
Welker, D. L. \& Williams, K. L. (1980b). The assignment of four new loci, including the coumarin sensitivity locus $\operatorname{cou} A$, to linkage group VII of Dictyostelium discoideum. J Gen Microbiol 120, 149-159.

Welker, D. L. \& Williams, K. L. (1982). A genetic map of Dictyostelium discoideum based on mitotic recombination. Genetics 102, 691-710.

Welker, D. L., De Lozanne, A. \& Spudich, J. A. (1989). Linkage analysis of the myosin heavy chain gene in Dictyostelium discoideum using a mutation generated by homologous recombination. Mol Gen Genet 216, 498-502.

Williams, J. G., Ceccarelli, A., McRobbie, S., Mahbubani, H., Kay, R. R., Early, A., Berks, M. \& Jermyn, K. A. (1987). Direct induction of Dictyostelium prestalk gene expression by DIF provides evidence that DIF is a morphogen. Cell 49, 185-192.

Williams, J. G., Duffy, K. T., Lane, D. P., McRobbie, S. J., Harwood,
A. J., Traynor, D., Kay, R. R. \& Jermyn, K. A. (1989). Origins of the prestalk-prespore pattern in Dictyostelium development. Cell 59, $1157-1163$.

Williams, K. L. \& Newell, P. C. (1976). A genetic study of aggregation in the cellular slime mould Dictyostelium discoideum using complementation analysis. Genetics 82, 287-307.

Williams, K. L., Kessin, R. H. \& Newell, P. C. (1974). Parasexual genetics in Dictyostelium discoideum: mitotic analysis of acriflavin resistance and growth in axenic medium. $J$ Gen Microbiol 84, 59-69.

Witke, W., Schleicher, M. \& Noegel, A. A. (1992). Redundancy in the microfilament system: abnormal development of Dictyostelium cells lacking two F-actin cross-linking proteins. Cell 68, 53-62.

Received 2 October 1995; accepted 12 December 1995. 